\title{
Adenoviral cardiotrophin-1 gene transfer protects pmn mice from progressive motor neuronopathy
}

\author{
Thierry Bordet, ${ }^{1}$ Henning Schmalbruch, ${ }^{2}$ Brigitte Pettmann, ${ }^{3}$ Albert Hagege, ${ }^{4}$ \\ Laetitia Castelnau-Ptakhine, ${ }^{1}$ Axel Kahn, ${ }^{1}$ and Georg Haase ${ }^{1,3}$
}

1'Institut National de la Santé et de la Recherche Médicale (INSERM) U.129, Institut Cochin de Génétique Moléculaire,
75014 Paris, France
${ }^{2}$ Institute of Medical Physiology, Panum Institute, University of Copenhagen, 2200 Copenhagen N, Denmark
${ }^{3}$ INSERM U.382, Institut de Biologie du Développement de Marseille, 13288 Marseille, France
${ }^{4}$ Laboratoire de Recherche en Imagerie, Faculté de Médecine Necker-Enfants Malades, 75015 Paris, France

Address correspondence to: Axel Kahn, INSERM U.129, Institut Cochin de Génétique Moléculaire, 24, rue du fb St. Jacques, 75014 Paris, France. Phone: 33-1-44-41-24-24; Fax: 33-1-44-41-24-21; E-mail: kahn@cochin.inserm.fr.

Received for publication January 11, 1999, and accepted in revised form September 10, 1999.

\begin{abstract}
Cardiotrophin-1 (CT-1), an IL-6-related cytokine, causes hypertrophy of cardiac myocytes and has pleiotropic effects on various other cell types, including motoneurons. Here, we analyzed systemic CT-1 effects in progressive motor neuronopathy $(\mathrm{pmn})$ mice that suffer from progressive motoneuronal degeneration, muscle paralysis, and premature death. Administration of an adenoviral CT-1 vector to newborn $p m n$ mice leads to sustained CT- 1 expression in the injected muscles and bloodstream, prolonged survival of animals, and improved motor functions. CT-1-treated pmn mice showed a significantly reduced degeneration of facial motoneuron cytons and phrenic nerve myelinated axons. The terminal innervation of skeletal muscle, grossly disturbed in untreated pmn mice, was almost completely preserved in CT-1-treated pmn mice. The remarkable neuroprotection conferred by CT-1 might become clinically relevant if CT-1 side effects, including cardiotoxicity, could be circumvented by a more targeted delivery of this cytokine to the nervous system.
\end{abstract}

J. Clin. Invest. 104:1077-1085 (1999).

\section{Introduction}

Human motor neuron diseases such as amyotrophic lateral sclerosis (ALS) and spinal muscular atrophy (SMA) result from degeneration of spinal neurons and, in ALS, also cortical motor neurons, leading to severe muscular atrophy. Mutations in the superoxide dismutase 1 (SOD1) gene have been identified in a subset of familial ALS, and deletions or mutations in the survival motor neuron gene are responsible for SMAs (reviewed in refs. 1, 2). Mechanisms of motoneuron degeneration, however, remain incompletely understood. In vitro and in vivo experiments have suggested that motoneuron diseases might benefit from neurotrophic factor administration (3-5). Indeed, brain-derived neurotrophic factor (BDNF), neurotrophin3 (NT-3), NT-4/5, ciliary neurotrophic factor (CNTF), and glial cell line-derived neurotrophic factor (GDNF) protect motoneurons from acute death induced by peripheral nerve axotomy in neonate rodents (6-10). Furthermore, CNTF, NT-3, and BDNF show protective effects on motoneurons in murine models of inherited progressive motoneuron degeneration such as progressive motor neuronopathy $(p m n)(11,12)$ and wobbler $(13,14)$. None of these factors, however, has prevented the onset or halted the course of the disease, which has prompted us to test other factors in motoneuron disease models.

We have chosen cardiotrophin-1 (CT-1), a member of the IL- 6 family of cytokines. Like leukemia inhibitory factor (LIF), CNTF, and oncostatin M (OSM), CT-1 signals via the LIFR $\beta / g p 130$ receptor complex (15) and a third specific receptor component $(\mathrm{CT}-1 \mathrm{R} \alpha)(16,17)$.
CT-1 was originally identified as a cardiac myocyte hypertrophic factor (18) but shares biological activities of the IL- 6 members, i.e., on hematopoietic cells, embryonic stem cells $(15,19)$, hepatocytes $(20,21)$, and neuronal cells $(15,22)$. In particular, CT- 1 is a very potent neurotrophic factor for spinal motoneurons in longterm culture and protects neonatal sciatic motoneurons from axotomy-induced cell death in rats (16). Furthermore, CT-1 may be a naturally relevant neurotrophic factor for developing motoneurons, as it is present at high levels in embryonic limb bud in vivo $(16,23)$.

Here, we have investigated the therapeutic potential of CT- 1 in the mouse mutant pmn. This mutant is characterized by a prominent axonal degeneration that starts at the endplates of motor nerves and leads to pelvic girdle and hindlimb atrophy, impaired motor performance, and ultimately death (24). Adenoviral vectors represent an interesting means to achieve high and stable delivery of neurotrophic factors in vivo $(12,25-27)$. In this study, we treated newborn pmn mice by intramuscular adenoviral gene transfer of CT-1 and observed an improvement in both functional and histological parameters.

\section{Methods}

Construction of the AdCT-1 recombinant adenoviral vector. Murine CT-1 cDNA was isolated by screening of an adult mouse muscle cDNA library with a PCR probe corresponding to nucleotides 504-785 of the mouse CT-1 cDNA (GenBank accession no. U18366). The CT-1 reading frame was fitted with the 60-bp preNGF leader 


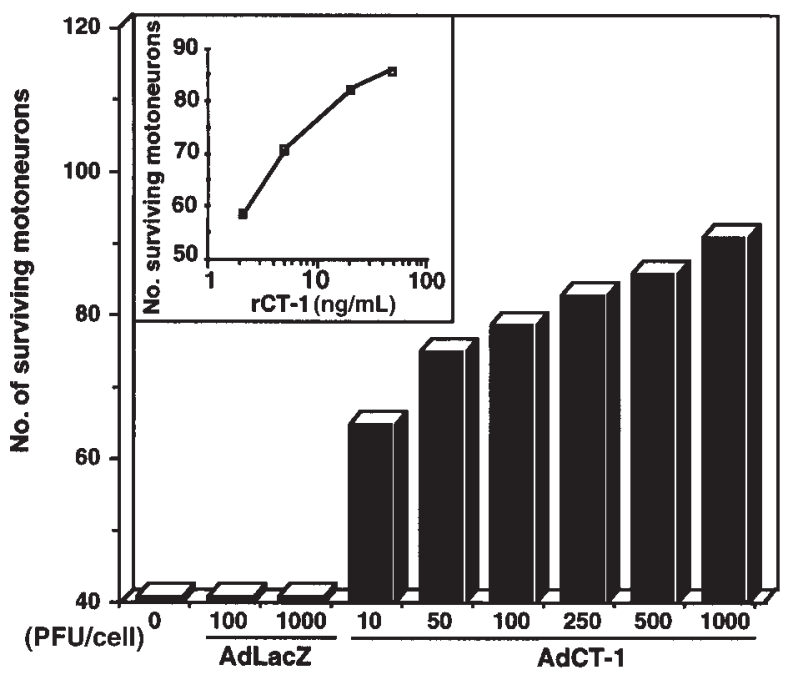

Figure 1

Adenovirus-mediated production of biologically active CT-1. Conditioned media from AdCT-1- or AdLacZ-infected human fibroblasts were tested for their trophic activity on cultured motoneurons. The number of surviving motoneurons was determined 48 hours after plating and is represented as a function of the number of infectious particles per fibroblast (PFU/cell) or as a function of recombinant CT-1 protein ( $\mathrm{rCT}-1$; inset). Each point represents the mean of duplicate plates.

sequence, the long terminal repeat of Rous sarcoma virus (RSV) and the SV40 polyadenylation signal. This expression cassette was subcloned in a shuttle plasmid in place of the E1 region of the adenoviral genome. The AdCT-1 vector was obtained by a 2 -step recombinational cloning in Escherichia coli, with the CT-1 shuttle plasmid and a replicon providing the adenoviral genome deleted in regions E1 and E3 (28). Adenoviral vectors were amplified and titered on 293 cells according to standard methods (29). Construction of the AdLacZ vector has been described previously (30).

Cell culture methods and in vitro assays. Human fibroblasts were grown to a density of $5 \times 10^{5}$ cells per $25-\mathrm{cm}^{2}$ dish. Adenoviral infection was carried out for 1 hour at different moi's, and conditioned media $(2 \mathrm{~mL})$ were sampled at from 24-48 hours after infection.

Motoneurons from ventral spinal cords of embryonic day 14.5 Sprague-Dawley rats were isolated essentially as described by Henderson et al. (31). Purified motoneurons were seeded on polyornithine/laminin-coated dishes at a density of 1,000 cells per 16-mm well. Samples (conditioned media, mouse sera, or recombinant murine CT-1 protein; kindly provided by Genentech, San Francisco, California, USA) were distributed in the coated well plates before cell seeding. After 48 hours in culture, motoneuron survival was quantified by counting the number of large phase-bright neurons with long axonal processes (16).

Ciliary ganglion neurons from 8-day-old chicken embryos were isolated and cultured as described by Manthorpe and Varon (32). Their survival was assessed in the presence of mouse sera or recombinant CT-1 protein as detailed previously (33).

Adenoviral vector injection in pmn mice. At 3-5 days of age, homozygous pmn mice, heterozygous $X t / p m n$ littermates
(34), and normal C57BL/6J mice were anesthetized by hypothermia and injected intramuscularly with $10^{7}$ to 3 $\times 10^{8}$ plaque-forming units (PFU) of virus in $100 \mu \mathrm{L}$ of PBS. The adenoviral suspension was injected into the right gastrocnemius $(50 \mu \mathrm{L})$, the right triceps brachii $(25$ $\mu \mathrm{L})$, and the long dorsal muscles of the thoracic trunk $(25 \mu \mathrm{L})$. Animal experimentation was performed in compliance with institutional guidelines.

Analysis of CT-1 expression in tissues by RT-PCR and Western blot. Gastrocnemius muscles and lumbar spinal cords mice were dissected and flash-frozen in liquid nitrogen. Total RNAs were isolated using the RNA-B reagent (Bioprobe, Montreuil, France) and reverse transcribed with the M-MLV RT (GIBCO BRL, Grand Island, New York, USA). RT-PCR analysis of adenoviral CT-1 transcripts were performed with primers in the RSV promoter (5'ACCATTCACCACATTGGTGT) and in CT- 1 cDNA (5'GCACGTATTCCTCCAGAAG). Control RT-PCR reactions were carried out using $\beta$-actin primers. Proteins were prepared by homogenization and sonication in $100 \mathrm{mM} 20 \%$ (wt/vol) potassium phosphate buffer ( $\mathrm{pH} 7.8$ ), $0.2 \%$ Triton X-100, and protease inhibitors; centrifugation for 30 minutes at 30,000 g; and collection of supernatants. CT- 1 immunoreactive protein was detected on Western blots with a rabbit polyclonal CT-1 antibody raised against recombinant murine CT-1 expressed in E. coli (Covalab, Oullins, France). A total of $150 \mu \mathrm{g}$ and $50 \mu \mathrm{g}$ of proteins was loaded on $12 \%$ SDS-PAGE to reveal endogenous and adenoviral CT-1 expression, respectively.

Histological examinations. Animals were deeply anesthetized with ether and transcardially perfused with glutaraldehyde. The distal phrenic nerves were postfixed with osmic tetroxide and embedded in epoxy resin. Myelinated fibers were counted in $p$-phenylenediamine-stained 3 - $\mu \mathrm{m}$-thick cross sections. The terminal motor innervation of thin abdominal muscles was stained by the AChE-silver method $(35,36)$. Digital images were taken with $\mathrm{a} \times 20$ objective, and the distances between last axon branching and endplate were measured with NIH Image (National Institutes of Health, Bethesda, Maryland, USA) and processed with KaleidaGraph software (Synergy Software, Reading, Pennsylvania, USA). Micrographs were reconstructed from through-focus series by means of the Auto-Montage software (Synoptics Ltd., Cambridge, United Kingdom).

Brains perfused with buffered formaldehyde were embedded in paraffin and $7-\mu \mathrm{m}$-thick serial sections of the brain stem were stained with cresyl violet. The nucleoli of facial motoneurons were counted in every fifth section on both sides without correction for split nucleoli (37).

Electrophysiology. The mice were lightly anesthetized with Fentanyl citrate fluanisone to suppress voluntary activity and pain reactions to needle insertion. A concentric needle electrode $(0.5 \mathrm{~mm}$ diameter) with a leadoff area of $0.03 \mathrm{~mm}^{2}$ was advanced through the lateral abdominal wall to the right diaphragm, and the electromyogram was recorded. Care was taken not to record from intercostal muscles and not to penetrate the diaphragm. For each mouse, 5-15 inspiratory bursts recorded from at least 2 sites were analyzed; duration, 


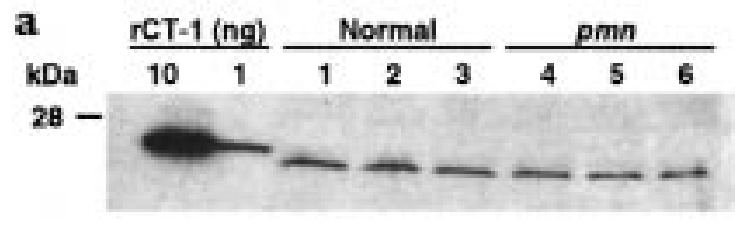

b
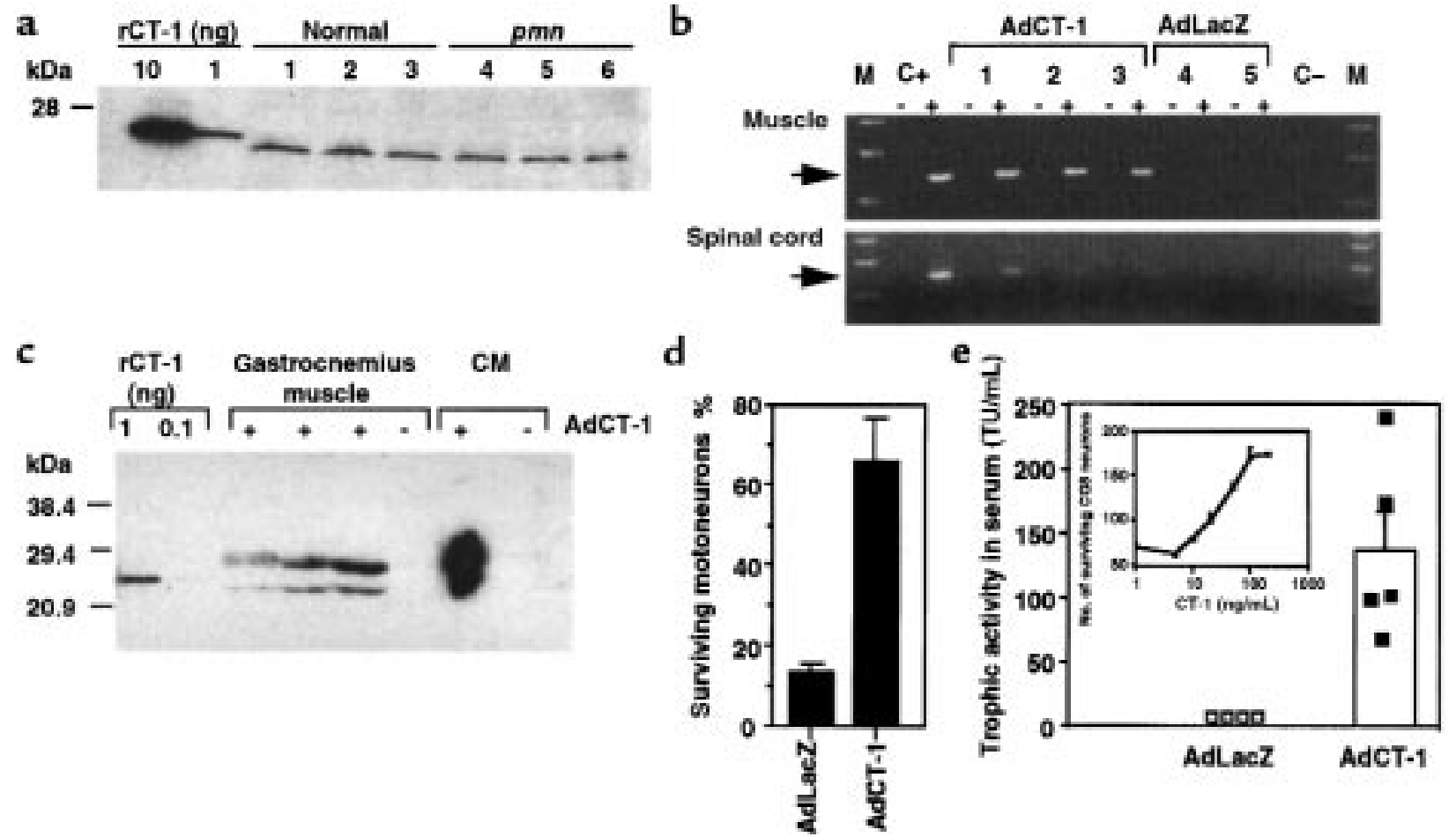

Figure 2

CT-1 gene expression in pmn mice. (a) Levels of endogenous CT-1 protein in gastrocnemius muscles of 28-day-old normal (lanes 1-3) and pmn (lanes 4-6) mice analyzed by Western blot. (b, c, d, and e) CT-1 expression after AdCT-1 intramuscular injection in neonatal pmn mice. (b) The right gastrocnemius muscle and the lumbar spinal cord were analyzed in AdCT-1-injected (lanes 1-3) and AdLacZ-injected (lanes 4-5) mice. Adenoviral CT1 transcripts were detected by RT-PCR in all right gastrocnemius muscles of AdCT-1-injected mice at day 25 , whereas only weak RT-PCR signals were detected in the lumbar spinal cords of 15-day-old treated mice. C+: cDNA from AdCT-1-infected 293 cells; C-: without template; -, without RT; +, with RT. (c) CT-1 overexpression was revealed by Western blot analysis in AdCT-1-injected muscles (+) but not in AdLacZ-injected muscle (-). Recombinant CT-1 protein ( $\mathrm{rCT}-1)$ and conditioned media (CM) from AdCT-1-infected (+) or noninfected (-) fibroblasts were used as controls. The 2 bands of apparent molecular mass 23 and $29 \mathrm{kDa}$ might reflect precursor and mature forms of CT-1 or differently glycosylated isoforms. Note that endogenous CT-1 protein in untreated pmn mice was not detectable under these experimental conditions (50 $\mu \mathrm{g}$ of loaded protein, short exposure time). (d) Sera of AdCT-1- and AdLacZ-injected pmn mice were tested for their neurotrophic activity on motoneurons. The number of motoneurons per field (mean \pm SEM) was determined and expressed relative to the number of motoneurons surviving in $50 \mathrm{ng} / \mathrm{mL} \mathrm{CT-1} \mathrm{(100 \% )} \mathrm{and} \mathrm{in} \mathrm{neurobasal}$ medium (0\%). (e) Sera of 25-day-old pmn mice and rCT-1 (inset) were tested in a ciliary ganglion (CG8) neuron survival assay. Elevated serum CT-1 concentrations (in trophic units $[\mathrm{TU}] / \mathrm{mL}$ ) were detected in all 5 tested AdCT-1-injected pmn mice but in none of the 4 AdLacZ-injected mice. One TU corresponds to the serum dilution that allowed half maximal survival of the neurons.

maximal amplitude, and number of negative spikes of at least $30 \mu \mathrm{V}$ amplitude were determined. The means for each mouse were computed, and differences were tested with Student's $t$ test.

Compound action potentials of the calf muscles were elicited by supramaximally stimulating the sciatic nerve at the sciatic notch through noninsulated platinum needle electrodes and were recorded between 2 electrodes placed over the belly of the anterior tibial muscle and at the ankle. Shape and amplitude of the potentials did not change when the recording electrode was moved to the gastrocnemius muscle; this indicated that the recorded potential was the combined extensor and flexor muscle potential. Peak-to-peak amplitudes and the motor latencies were measured. All stimulations and recordings were done with a KEYPOINT electromyograph (Dantec, Copenhagen, Denmark).

Echocardiographic methods. Cardiac function and structure in anesthetized mice were studied by 2-dimensional and M-mode echocardiography (Sequoia; Acuson, Mountain Valley, California, USA) using a short-focus
13- to $15-\mathrm{Mhz}$ linear phased-array transducer $(15 \mathrm{~L} 8$; Acuson). The heart was first imaged in the 2-dimensional parasternal long-axis view of the left ventricle (LV) ; the M-mode cursor was then positioned perpendicular to the septum and posterior wall just below the tip of the mitral valve leaflets. Only M-mode tracings with welldefined continuous interfaces were accepted for linear dimensions measurements. All measurements were made online, at a speed of $200 \mathrm{~mm} / \mathrm{s}$ from images captured on cineloops, independent of the electrocardiogram (ECG) and using the American Society of Echocardiography leading edge method (38). The investigator (A. Hagege) was blinded to the treatment status of the mice; measurements were averaged on 3-5 consecutive cycles.

\section{Results}

Biological activity of CT-1 expressed by adenoviral vector. We constructed a replication-defective adenoviral vector (AdCT-1) coding for murine CT-1. As CT-1 does not contain a hydrophobic $\mathrm{NH}_{2}$-terminal leader sequence characteristic of secreted proteins (18), the CT-1 cDNA was 
Figure 3

Electromyography in 35-day-old

AdCT-1-treated pmn mice. (a) Individual and mean amplitude of CMAPs and motor latency in the calf muscles of normal, untreated pmn, and ACT-1-treated pmn mice. Data of untreated pmn and AdCT-1-treated $p m n$ mice were significantly different $\left({ }^{*} P<0.05\right)$. (b) Spontaneous electrical activity in the right diaphragm. Note the differences in shape of the inspiratory burst and the frequency of the high-voltage oscillations between untreated and CT-1-treated pmn mice. Asterisks mark ECG recordings.

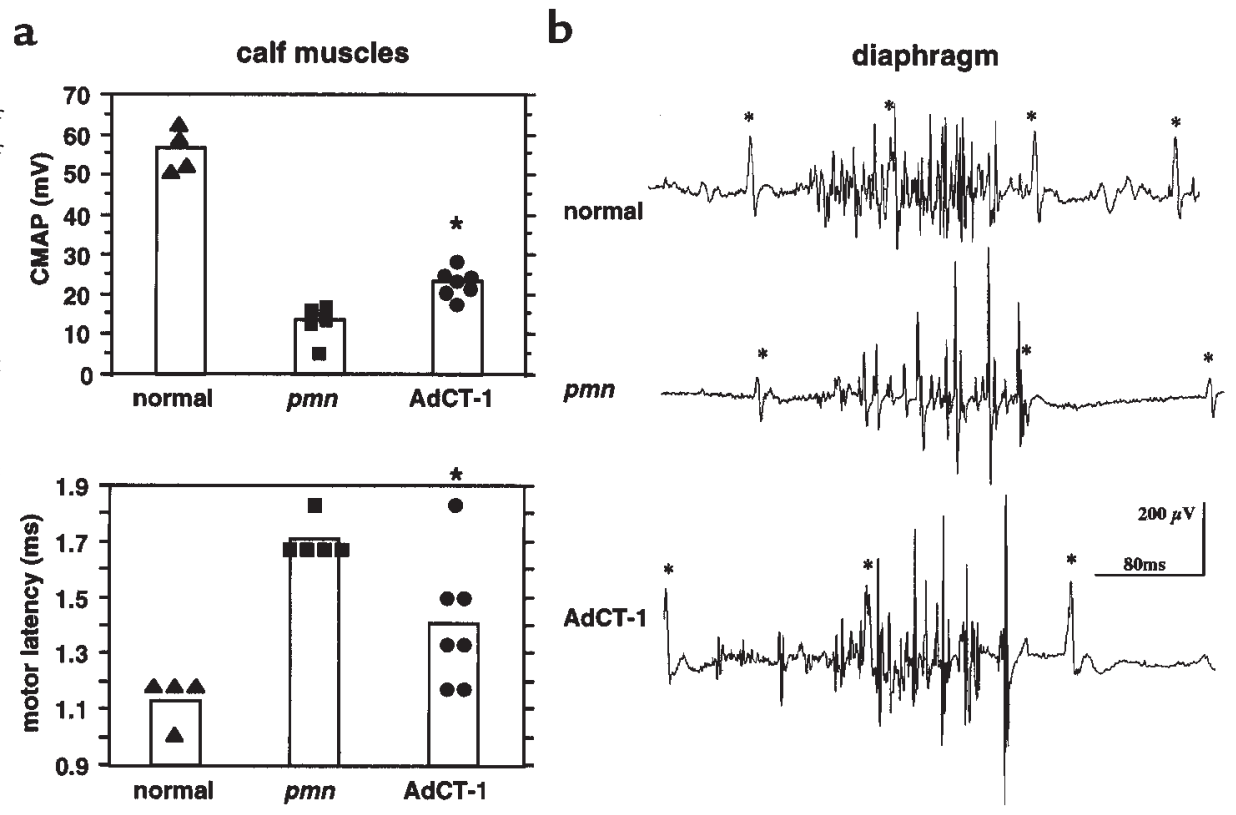

placed behind the mouse $\beta$ NGF leader sequence. To assess the biological activity of this vector, human fibroblasts were infected with AdCT-1 at different moi's, and their conditioned media were tested in a motoneuron survival assay (Figure 1). A dose-dependent increase in motoneuron survival was observed in conditioned media after AdCT-1 but not after AdLacZ infection. At the optimal titer (1,000 PFU/cell), adenoviral production of biologically active CT- 1 was estimated to be $140 \mathrm{ng}$ per million cells per 24 hours, as calculated from the dose-response curve established with recombinant CT-1 protein.

Endogenous CT-1 expression in pmn mice. It has been hypothesized that a lack of target-derived neurotrophic factors might contribute to or even be responsible for motoneuronal degeneration in ALS (39) and in pmn mice (40). However, in gastrocnemius muscles of $p m n$ mice and normal littermates, we measured similar amounts of CT- 1 mRNA by competitive RT-PCR (data not shown) and CT- 1 protein by Western blot (Figure $2 \mathrm{a}$ ). Because CT-1 mRNA and protein levels are normal in pmn mice, the intended adenoviral CT-1 delivery in pmn mice can be regarded as a pharmacological treatment.

Intramuscular AdCT-1 gene transfer into pmn mice. Neonatal (3- to 5-day-old) pmn mice were injected with $10^{8} \mathrm{PFU}$ of AdCT-1 into the right gastrocnemius, triceps brachii, and the long muscles of the thoracic trunk. At day 25, adenoviral CT-1 transcripts were detected by RT-PCR in the right gastrocnemius muscles from all treated mice (Figure 2b). Weak RT-PCR signals were also detected in the lumbar spinal cords of treated mice aged 15 days. At day 25 , a high level of CT-1 protein was detected in muscle extracts by immunoblotting (Figure 2c). Using the motoneuron survival assay, we also showed that the sera of AdCT-1-treated mice had a serum trophic activity significantly above the background in sera of AdLacZinjected mice (Figure $2 \mathrm{~d}$ ). In the absence of an available ELISA for CT-1, we used a chicken ciliary ganglion neuron survival assay to determine more accurately the cir- culating CT-1 levels (Figure 2e). A mean of $137 \pm 31$ trophic $\mathrm{U} / \mathrm{mL}$ serum was measured in 25 -day-old AdCT1-injected pmn mice, whereas no circulating CT-1 bioactivities could be detected in AdLacZ-injected pmn mice.

Improved motor functions in CT-1 treated pmn mice. AdCT1 did not delay the usual onset of muscle weakness and wasting around day 15 , but progression was slower than in untreated pmn mice. To assess the innervation of the early affected hindlimb muscles, we recorded the amplitude of the evoked motor response (compound muscle action potential [CMAP]) of the calf muscles after supramaximal electrical stimulation of the sciatic nerve (Figure 3a). At 35 days of age, the amplitude of the CMAP in untreated pmn mice was $13 \pm 2 \mathrm{mV}(n=5)$, corresponding to only $20 \%$ of the normal $(58 \pm 4 \mathrm{mV} ; n=4)$. After AdCT-1 treatment, the evoked response in $p m n$ mice was almost twice as large $(23 \pm 1 \mathrm{mV} ; n=7 ; P<0.01)$. Also, the motor latency was shorter in AdCT-1-treated $(1.4 \pm 0.08 \mathrm{~ms} ; n=7)$ than in untreated $p m n$ mice $(1.7 \pm$ $0.03 \mathrm{~ms} ; n=5 ; P<0.05)$. In normal mice of the same age, the latency was $1.1 \pm 0.04 \mathrm{~ms}$.

Because $p m n$ mice die from respiratory insufficiency with acidosis and hypercapnia, we investigated the electromyogram of the diaphragm. The number and the mean amplitude of the positive-negative deflections (turns) are indicators of the number of motor unit potentials during an inspiratory burst and of the size of the motor units recruited. Representative recordings from 35 -day-old mice are shown in Figure 3b. The inspiratory bursts in normal mice consisted of regular and high-frequency spikes that were irregular and less frequent in untreated pmn mice owing to ongoing denervation of the motor units. Although the number of negative spikes increased in AdCT-1-treated mice $(29 \pm 3.3$ turns vs. $17 \pm$ 3.4 in AdLacZ-injected mice; $P=0.03$ ), their amplitudes $(580 \pm 130 \mu \mathrm{V}$ and $520 \pm 211$, respectively) did not differ. This suggests that after CT- 1 treatment, either more motor units were recruited during the inspiratory phase 
or they discharged at a higher frequency, whereas the size of the motor units had not increased by collateral reinnervation.

Terminal innervation pattern in AdCT-1-treated pmn mice. To assess whether CT-1 improved neuromuscular functions by delaying nerve degeneration rather than by inducing collateral reinnervation, we examined the terminal motor innervation pattern in CT-1-treated mice by using the acetylcholinesterase-silver method. Terminal axons in abdominal muscles of normal mice usually supplied 1 motor endplate, and most of them ran without branching for 100-400 $\mu \mathrm{m}$ (Figure 4, a and e). They were shorter and often interrupted in untreated pmn mice, and many endplates lacked an axon, i.e., were denervated (Figure 4, b and e). Nodal and preterminal sprouts were frequent but usually thin and blind ending. In contrast, muscles of AdCT-1-treated pmn mice (Figure 4, c-e) contained few denervated endplates. Instances of obvious reinnervation by terminal, preterminal, or nodal sprouts (Figure 4c) were rare, which is in agreement with the electrophysiology of the diaphragm. Terminal axons were longer after CT-1 treatment than in untreated pmn but shorter than in normal mice (Figure 4e).

Protective effects of CT-1 against degeneration of phrenic nerve axons and facial motoneurons. The phrenic nerve is severely degenerated in pmn mice with pronounced loss of

Table 1
Number of phrenic myelinated axons and facial motoneurons in pmn mice after AdCT-1 treatment (mean \pm SEM)

\begin{tabular}{|c|c|c|c|}
\hline \multicolumn{2}{|l|}{ Group } & $\begin{array}{l}\text { Phrenic axons } \\
\quad(\text { day 25) }\end{array}$ & $\begin{array}{l}\text { Facial motoneurons } \\
\text { (day 35) }\end{array}$ \\
\hline \multicolumn{2}{|l|}{ Normal mice } & $263 \pm 8(n=4)$ & $3,132 \pm 94(n=3)$ \\
\hline \multicolumn{2}{|l|}{ Untreated pmn mice } & $122 \pm 13(n=8)$ & $2,193 \pm 64(n=3)$ \\
\hline \multicolumn{2}{|l|}{ AdLacZ-injected pmn mice } & $120 \pm 11(n=6)$ & ND \\
\hline \multirow[t]{3}{*}{ AdCT-1-treated pmn mice } & $10^{8} \mathrm{PFU}$ & $197 \pm 12(n=12)^{\mathrm{A}}$ & $2,587 \pm 45(n=4)^{\mathrm{B}}$ \\
\hline & $5 \times 10^{7} \mathrm{PFU}$ & $144 \pm 15(n=9)$ & ND \\
\hline & $10^{7} \mathrm{PFU}$ & $134 \pm 12(n=6)$ & ND \\
\hline
\end{tabular}

A,BStudent's $t$ test: $P<0.001$ and $P=0.01$, respectively. ND, not determined.

myelinated fibers (24). After CT-1 treatment, the nerve looked surprisingly intact (Figure 5). At day 25, the phrenic nerves of AdCT-1-treated pmn mice contained $50 \%$ more myelinated fibers than control pmn mice $(P<$ 0.0001; Table 1). Even at 45 days, when most untreated pmn mice had already died, the fiber number in treated mice was still relatively high $(160 \pm 8$ axons; $n=10)$. This effect of CT-1 was dose-dependent (Table 1).

We also examined whether CT-1 is able to protect motoneuron cell bodies. As reported previously $(11,41)$, the facial nucleus of 4- to 6-week-old pmn mice shows significant degeneration and loss of motoneurons. We performed serial sections from the brain stem of 35-dayold mice and showed that the number of facial
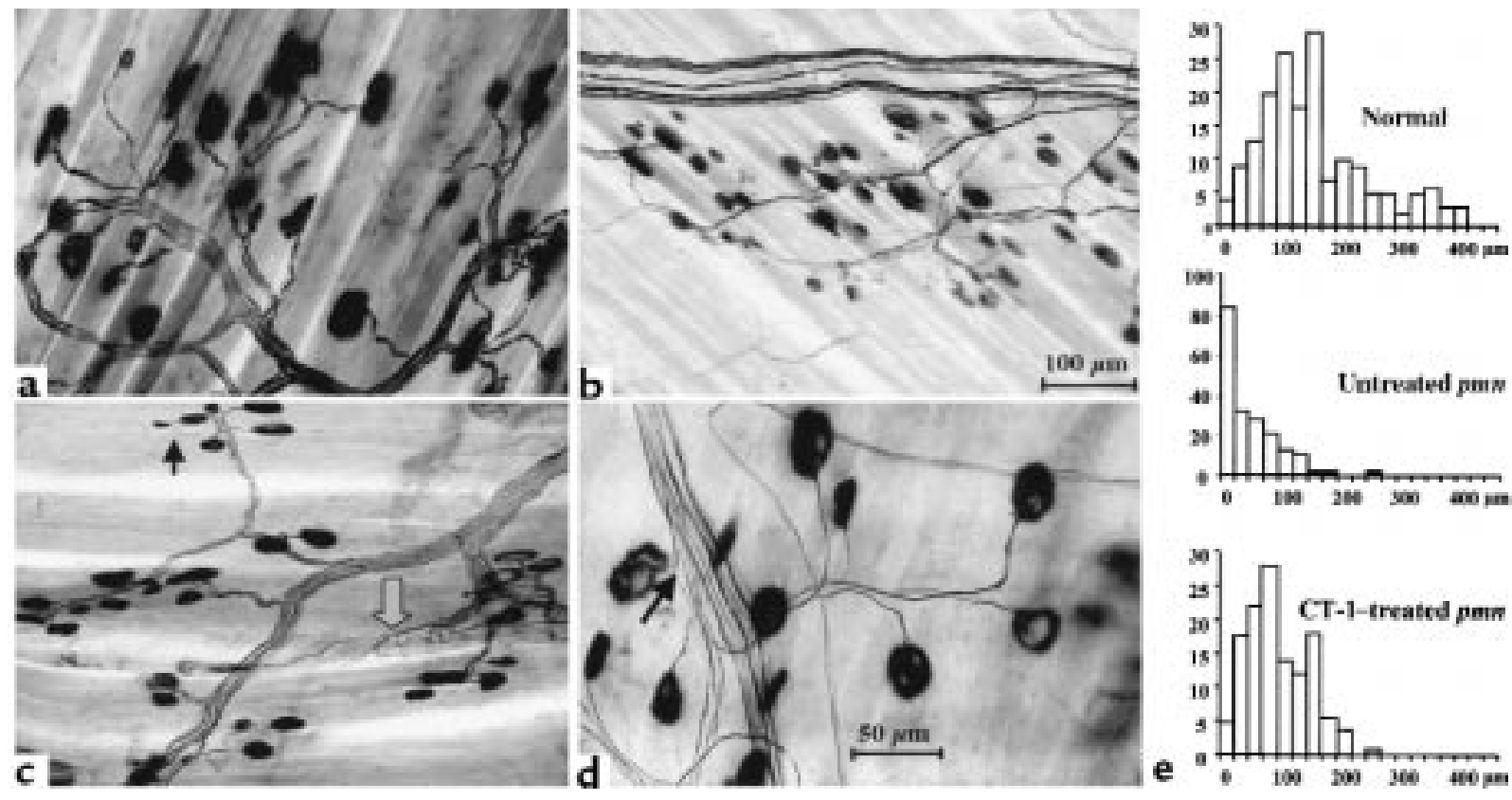

Figure 4

Terminal innervation of abdominal muscles of 4-week-old mice, stained with the AchE-silver method. (a) Normal mouse (Xt/pmn). Bundles of terminal axons originating from intramuscular nerve branches supply individual endplates. These axons run without branching for up to $400 \mu \mathrm{m}$. (b) pmn mouse, untreated. A nerve branch contains few intact, possibly afferent fibers and remnants of degenerated axons. Many endplates are devoid of terminal axons. Between these endplates are numerous fine axonal sprouts that, however, are not visible at this magnification. (c and $\mathbf{d})$ pmn mice, AdCT-1-treated. The nerve branch in $\mathbf{c}$ appears well preserved, and all endplates are supplied by relatively long terminal axons. Rare examples of unequivocal sprouting are marked in both micrographs (arrows). A muscle spindle is visible in c (open arrow). Calibration bars: $100 \mu \mathrm{m}$ for a-c, 50 $\mu \mathrm{m}$ for $\mathbf{d}$. (e) Histograms of the lengths of terminal axons of individual endplates measured from the last axonal branching to the entry into the endplate. The lengths of axons of denervated endplates (example of endplates without terminal axon) were recorded as zero. Note large number of denervated endplates in untreated pmn as compared with CT-1-treated pmn and normal mice. 


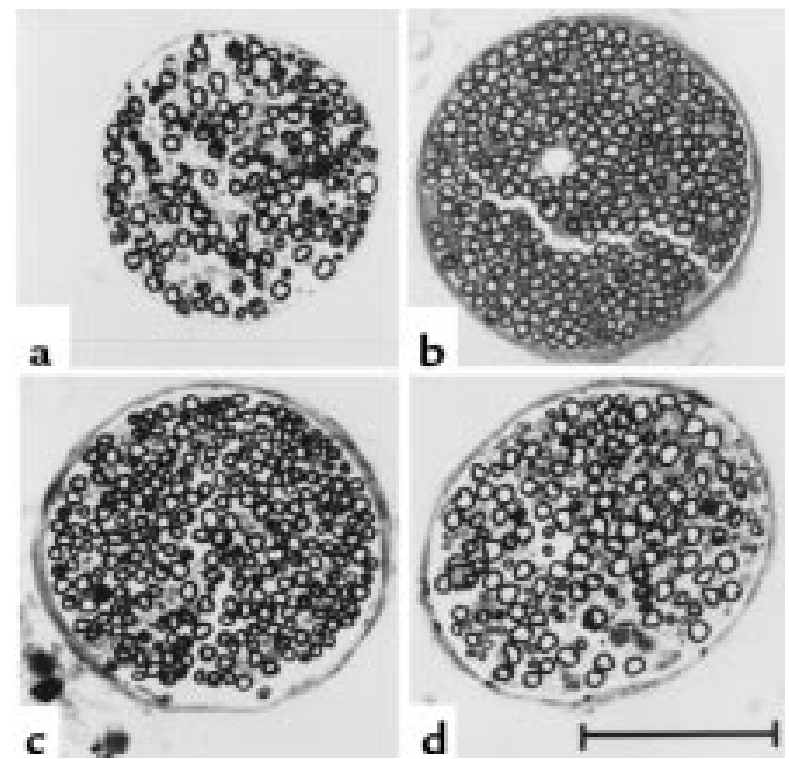

Figure 5

Light micrographs of phrenic nerves from 25-day-old (a, b, and $\mathbf{c}$ ) and 45-day-old (d) mice. (a) Untreated pmn; (b) normal littermate; (c and d) AdCT-1-treated pmn. Note the well-preserved nerve of the AdCT-1-treated mice at day 25 and still at day 45 compared with that of the 25-dayold untreated pmn mouse. Scale bar: $50 \mu \mathrm{m}$.

motoneurons was significantly increased in AdCT1 -treated $p m n$ mice compared with untreated $p m n$ mice (Figure 6 and Table 1).

Improved survival of CT-1 treated pmn mice. Altogether, the neuroprotective effects of the AdCT-1 injection resulted in an improved survival (Figure 7a). The AdCT-1-treated pmn mice survived for up to 67 days, with a mean of 48.2 \pm 1.6 days, which corresponds to an $18 \%$ increase in mean life-span when compared with AdLacZ-injected pmn mice $(40.8 \pm 1.6$ days; $\log$ rank $P=0.0012)$. At day $50,44 \%$ of AdCT-1-treated $p m n$ mice were still alive in comparison to only $10 \%$ of AdLacZ-injected pmn mice.

We also asked whether the survival of pmn mice might be further prolonged by higher AdCT-1 doses or by its combination with other neurotrophic factors. A higher dose of AdCT-1 (3 $\times 10^{8} \mathrm{PFU} /$ animal) failed to improve the mean survival and induced a significant decrease in weight gain (Figure $7 \mathrm{~b}$ ). Coinjection of AdCT-1 with $10^{9} \mathrm{PFU}$ of an ade-

Table 2

Cardiac functions in CT-1-treated C57BL/6J mice

$\begin{array}{lcc} & \text { Control } & \text { CT-1 } \\ \text { Group } & 482 \pm 16 & 474 \pm 10 \\ \text { HR (bmp) } & 3.2 \pm 1.3 & 3.4 \pm 0.9 \\ \text { LVDD }(\mathrm{mm}) & 1.7 \pm 1.8 & 2.1 \pm 1.3 \\ \text { LVSD }(\mathrm{mm}) & 34.6 \pm 4 & 40.4 \pm 3 \\ \text { LVDV }\left(\mathrm{mm}^{3}\right) & 5.8 \pm 2 & 9.3 \pm 2 \\ \text { LVSV }\left(\mathrm{mm}^{3}\right) & 0.63 \pm 0.02 & 0.53 \pm 0.03^{\mathrm{A}} \\ \text { Septal thickness }(\mathrm{mm}) & 0.57 \pm 0.01 & 0.52 \pm 0.04 \\ \text { Posterior wall thickness }(\mathrm{mm}) & 46.6 \pm 3 & 39.6 \pm 3 \\ \text { FS }(\%) & 84.2 \pm 2.8 & 77.2 \pm 3.2 \\ \text { EF }(\%) & \end{array}$

AStudent's $t$ test: $P=0.04$. Animal number was 4 in the control group and 6 in the $C T-1$ group. HR, heart rate; LVDD, maximal LV dimension in diastole; LVSD, minimal LV dimension in systole; LVDV, LV diastolic volume; LVSV, LV systolic volume; $\mathrm{FS}$, fractional shortening calculated as (LVDD - LVSD)/LVDD; EF, ejection fraction. noviral vector coding for GDNF (kindly provided by $\mathrm{P}$. Horellou and J. Mallet, Paris, France) also did not result in prolonged survival (mean: $49.3 \pm 2.4$ days; $n=12$ ).

These limitations prompted us to examine potential side effects of CT-1 that might have counterbalanced its neuroprotective effects. Given that injection of recombinant CT-1 protein can induce cardiotoxic effects in mouse (19), we investigated cardiac function by ECG methods. In this experiment, normal C57BL/6J mice rather than $p m n$ mice were studied because the small size of pmn mice precluded the use of the echographic probe. Neonate C57BL/6J mice were treated with the AdCT-1 vector as described previously and analyzed at 50 days of age. In AdCT-1-injected mice, LV volumes tended to be higher, whereas myocardium thickness was significantly lower, than in untreated mice (Table 2). Furthermore, the LV contractility (fractional shortening and ejection fraction) tended to be reduced after AdCT-1 treatment, but these differences did not reach statistical significance.

\section{Discussion}

CT-1, one of the most recently discovered neurotrophic factors, has demonstrated potent survival-promoting activities on motoneurons in vitro and after axotomy $(16,42)$. Our study is, to our knowledge, the first direct demonstration that CT-1, when delivered in vivo, has a relevant neurotrophic action in an animal model of motoneuron disease. After intramuscular gene transfer of an adenoviral vector encoding CT-1 into neonate $p m n$ mice, we measured high amounts of recombinant CT-1 mRNA and protein in the injected muscles and elevated CT-1 bioactivities in serum. Adenoviral CT-1 expression was also detected by RT-PCR in the spinal cord, in line with the previously reported retrograde transport of adenovirus in $\alpha$-motoneurons $(27,43,44)$. The levels of adenoviral CT-1 mRNAs in the lumbar spinal cords, however, were low, probably owing to the perturbation of axonal transport in pmn mice (45). We therefore assume that the therapeutic benefit of CT- 1 in pmn mice was mostly due to its systemic release from the infected muscles into the bloodstream.

Motoneurons in untreated pmn mice degenerate in a dying back process that first affects nerve terminals, axons, and only later their cytons. Motoneuron cytons have been shown to degenerate in the facial nucleus (11) but are preserved in the anterior horn of the lumbar spinal cord until late ages $(12,24)$. In this study, we report CT-1 actions in different territories and at various levels of the motoneuron. The AdCT-1 treatment rescued 42\% of the facial motoneurons, suggesting that peripheral CT1 can efficiently reach and protect these neurons in the facial nucleus of pmn mice like CNTF and GDNF (11, 41, 46). The denervation of the diaphragm presumably determines the course of the disease in pmn mice. Here, CT- 1 reduced by $56 \%$ the loss of myelinated fibers in the phrenic nerve of 25-day-old pmn mice, which represents the best result ever obtained in this mutant. Strong CT-1 neuroprotective effects were also seen at later ages, in line with the described long-term effects of CT-1 on spinal motoneurons in culture or after axotomy (16). Muscles innervated by the sciatic nerve are early affected by pmn. After CT-1 treatment, more muscle fibers retained their 

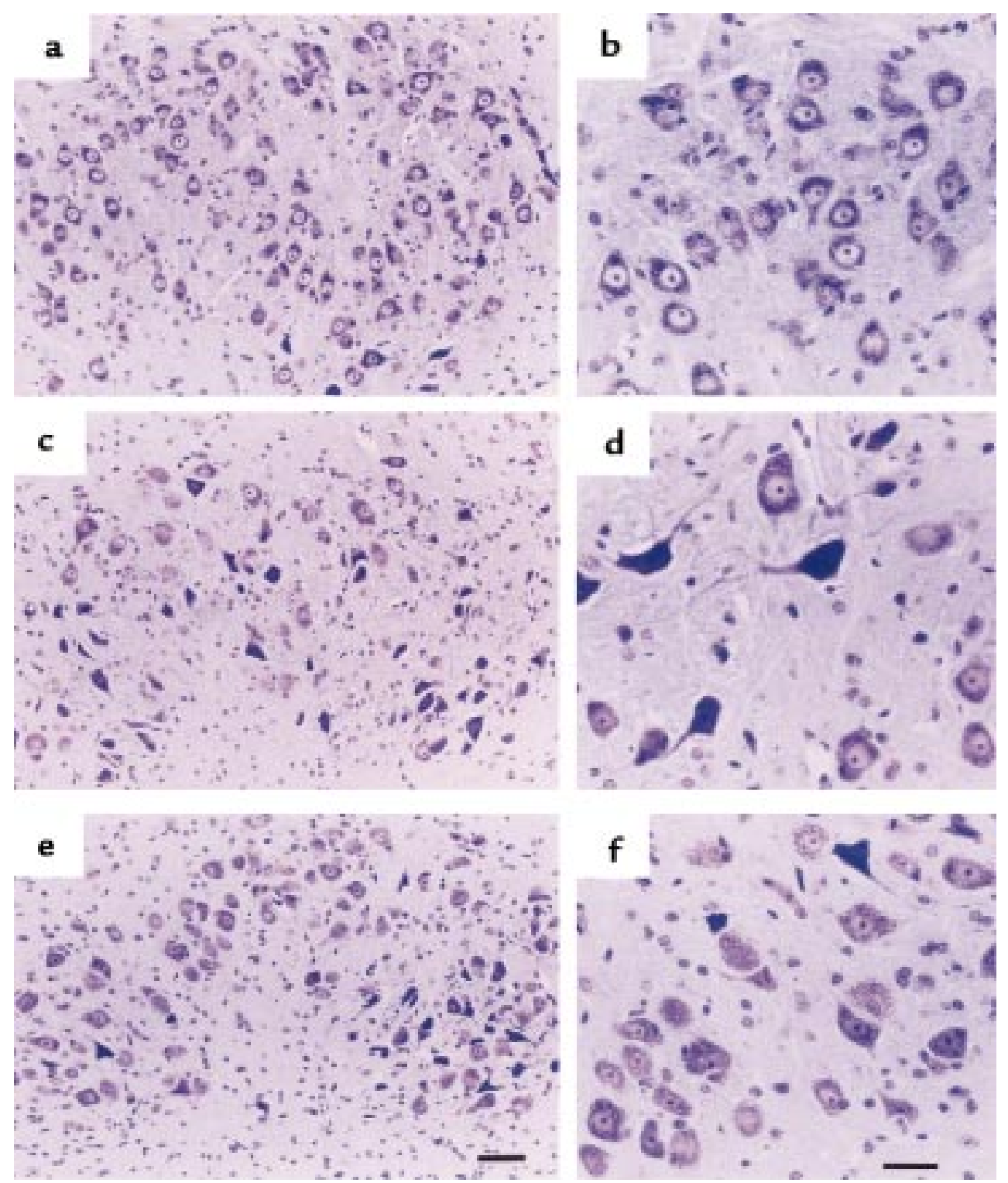

Figure 6

Light micrographs of facial nucleus of 35-day-old normal ( $\mathbf{a}$ and $\mathbf{b}$ ), untreated pmn (c and $\mathbf{d}$ ), and AdCT-1-treated pmn (e and $\mathbf{f})$ mice. In untreated pmn mice, the number of facial motoneurons was significantly decreased, and many of them showed extensive chromatolysis. Motoneurons were more numerous in the facial nucleus of AdCT-1-treated mice compared with untreated pmn mice (see also Table 1). Scale bar: $100 \mu \mathrm{m}$ (a, $\mathbf{c}$, and $\mathbf{e}$ ), and $50 \mu \mathrm{m}(\mathbf{b}, \mathbf{d}$, and $\mathbf{f})$.

innervation, allowing a slower decrease in the evoked action potential of the calf than in untreated pmn mice. Furthermore, nerve conduction velocity or neuromuscular transmission, or both, were improved, as evidenced by shorter latencies. Finally, the electrophysiological analysis of the inspiratory activity of the diaphragm provided evidence that CT-1 prevented degeneration and loss of motor units rather than that it promoted collateral reinnervation. This assumption was clearly strengthened by the near-normal appearance of the terminal innervation of abdominal muscles as compared with untreated pmn mice. Nevertheless, the terminal axons were shorter in CT-1-treated pmn mice than in normal mice. This might result from either preferential loss of the longest axons or successful nodal sprouting.
Despite these remarkable neuroprotective effects of CT-1, the life-span of AdCT-1-treated pmn mice was only modestly improved, by a mean of $18 \%$. This finding was particularly intriguing given the fact that the administration of adenoviral vectors coding for NT-3 or CNTF, while reducing the loss of phrenic myelinated fibers by about $30 \%$, resulted in, respectively, a $50 \%$ and $28 \%$ increase in the animals' survival $(12,33)$. Different explanations that are not mutually exclusive might account for this apparent discrepancy. First, it is obviously very difficult to compare the intrinsic efficacy of different neurotrophic factors after adenovirusmediated delivery in vivo because the blood concentrations may vary. On the other hand, one might argue that the serum CT-1 bioactivities reported in this study 
Figure 7

Survival and weight gain of pmn mice after AdCT1 gene transfer. (a) Survival of AdCT-1-treated mice (filled triangles; $n=18$ ) is significantly improved (log rank test, $P=0.0012$ ) compared with AdLacZ-injected mice (filled squares; $n=20$ ). (b) A dose-dependent reduction in weight gain was observed in AdCT-1-injected pmn mice (filled triangles, $10^{8} \mathrm{PFU}$; open triangles, $3 \times 10^{8} \mathrm{PFU}$ ) compared with AdLacZ-injected mice (filled squares, $\left.10^{8} \mathrm{PFU}\right)$.
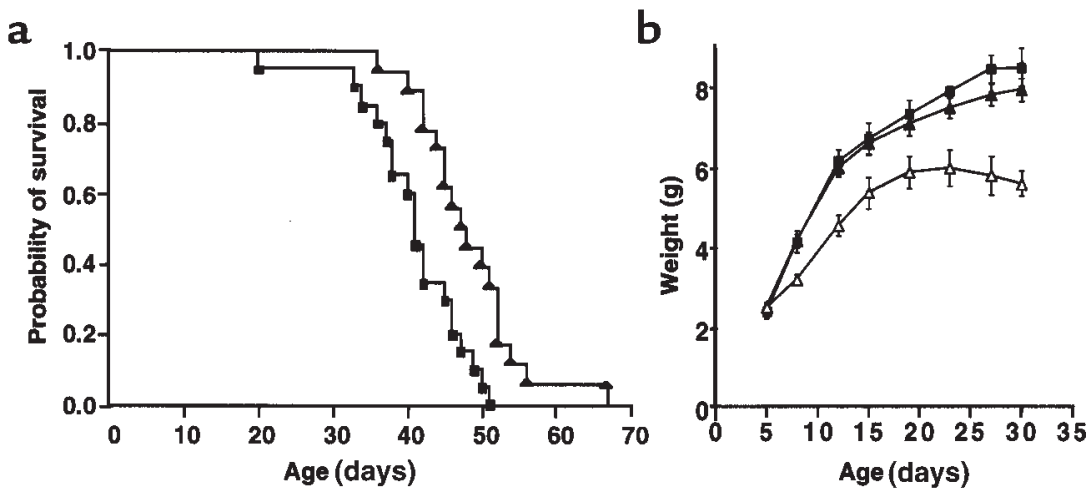

were in the same range as those of the previously tested cytokine CNTF (33). Second, CT-1 alone might not be sufficient to save a motoneuron subpopulation affected in pmn mice that might be critical for their survival. We therefore tested the coadministration of CT1 with GDNF, a neurotrophic factor belonging to the TGFb superfamily that can synergize with CT-1 to promote the survival of motoneurons in culture (42). The combined injection of both AdCT-1 and AdGDNF vectors, however, did not improve the life-span of pmn mice. In the future, combinations of CT-1 with neurotrophins such as NT-3 and NT-4/5 or IGF-1, which can all efficiently promote sprouting (47-49), might be more promising. Third, CT-1 side effects might have been induced by the AdCT-1 treatment. Indeed, we observed a reduction in weight gain and a dilatation of the left cardiac ventricle in AdCT-1-treated animals. Weight loss, proinflammatory responses, and induction of acute-phase proteins can be caused by several cytokines and have been extensively described after CNTF injection $(21,50-52)$. The stigmates of dilated cardiopathies that we observed in AdCT-1-treated normal mice are consistent with the morphological changes of cardiomyocytes cultured in the presence of CT-1 (53) and support the assumption that the neuroprotective effects of CT- 1 in pmn mice might have been counterbalanced by its systemic toxicity. Thus, we propose that the therapeutic index of CT- 1 might be increased by a more targeted delivery into the vicinity of motoneurons.

In conclusion, when delivered by adenoviral gene transfer into muscle of pmn mice, CT- 1 was a very potent factor in preventing motoneuron cell death and longterm motor axonal degeneration. CT- 1 inhibited the prominent muscle denervation observed in $p m n$ mice and improved neuromuscular functions. Because CT-1 is a cytokine with actions in various tissues, its therapeutic index in motoneuronal degeneration syndromes might be increased by targeting its actions to the motor unit or by its combination with other factors.

\section{Acknowledgments}

We thank M. Bjaerg for help in the histological analyses, J. Crouzet and L. Ferrero for advice in constructing the recombinant adenoviral vector, and J. Sanes for critical comments on the manuscript. This work was supported by grants from INSERM and the Association Française contre les Myopathies (AFM). T. Bordet is the recipient of an AFM fellowship.

1. Siddique, T., and Deng, H.X. 1996. Genetics of amyotrophic lateral sclerosis. Hum. Mol. Genet. 5:1465-1470.

2. Lefebvre, S., Burglen, L., Frezal, J., Munnich, A., and Melki, J. 1998. The role of the SMN gene in proximal spinal muscular atrophy. Hum. Mol. Genet. 7:1531-1536.

3. Thoenen, H., Hughes, R.A., and Sendtner, M. 1993. Trophic support of motoneurons: physiological, pathophysiological, and therapeutic implications. Exp. Neurol. 124:47-55.

4. Lindsay, R.M., Wiegand, S.J., Altar, C.A., and DiStefano, P.S. 1994. Neurotrophic factors: from molecule to man. Trends Neurosci. 17:182-190.

5. Henderson, C.E. 1995. Neurotrophic factors as therapeutic agents in ALS. Adv. Neurol. 68:235-240.

6. Yan, Q., Elliott, J., and Snider, W.D. 1992. Brain-derived neurotrophic factor rescues spinal motor neurons from axotomy-induced cell death. Nature. 360:753-755.

7. Sendtner, M., Holtmann, B., Kolbeck, R., Thoenen, H., and Barde, Y.-A. 1992. Brain-derived neurotrophic factor prevents the death of motoneurons in newborn rats after nerve section. Nature. 360:757-759.

8. Schmalbruch, H., and Rosenthal, A. 1995. Neurotrophin-4/5 postpones the death of injured spinal motoneurons in newborn rats. Brain Res. 700:254-260.

9. Sendtner, M., Kreutzberg, G.W., and Thoenen, H. 1990. Ciliary neurotrophic factor prevents the degeneration of motor neurons after axotomy. Nature. 345:440-441.

10. Henderson, C.E., et al. 1994. GDNF: a potent survival factor for motoneurons present in peripheral nerve and muscle. Science. 266:1062-1064

11. Sendtner, M., et al. 1992. Ciliary neurotrophic factor prevents degeneration of motor neurons in mouse mutant progressive motor neuronopathy. Nature. 358:502-504.

12. Haase, G., et al. 1997. Gene therapy of murine motor neuron disease using adenoviral vectors for neurotrophic factors. Nat. Med. 3:429-436.

13. Mitsumoto, H., et al. 1994. The effects of ciliary neurotrophic factor on motor neuron dysfunction in wobbler mouse motor neuron disease. Ann. Neurol. 36:142-148.

14. Mitsumoto, H., et al. 1994. Arrest of motor neuron disease in wobbler mice cotreated with CNTF and BDNF. Science. 265:1107-1110.

15. Pennica, D., et al. 1995. Cardiotrophin-1, biological activities and binding to leukemia inhibitory factor receptor/gp130 signaling complex. J. Biol. Chem. 270:10915-10922.

16. Pennica, D., et al. 1996. Cardiotrophin-1, a cytokine present in embryonic muscle, supports long-term survival of spinal motoneurons. Neuron. 17:63-74.

17. Robledo, O., et al. 1997. Signaling of the cardiotrophin-1 receptor, evidence for a third receptor component. J. Biol. Chem. 272:4855-4863.

18. Pennica, D., et al. 1995. Expression cloning of cardiotrophin 1, a cytokine that induces cardiac myocyte hypertrophy. Proc. Natl. Acad. Sci. USA. 92:1142-1146

19. Jin, H., et al. 1996. In vivo effects of cardiotrophin-1. Cytokine. 8:920-926.

20. Peters, M., Roeb, E., Pennica, D., Zumbuschenfelde, K.H.M., and Rosejohn, S. 1995. A new hepatocyte stimulating factor: cardiotrophin-1 (CT1). FEBS Lett. 372:177-180.

21. Richards, C.D., Langdon, C., Pennica, D., and Gauldie, J. 1996. Murine cardiotrophin-1 stimulates the acute-phase response in rat hepatocytes and H35 hepatoma cells. J. Interferon Cytokine Res. 16:69-75. 
22. Horton, A.R., Bartlett, P.F., Pennica, D., and Davies, A.M. 1998. Cytokines promote the survival of mouse cranial sensory neurones at different developmental stages. Eur. J. Neurosci. 10:673-679.

23. Sheng, Z., Pennica, D., Wood, W.I., and Chien, K.R. 1996. Cardiotrophin1 displays early expression in the murine heart tube and promotes cardiac myocyte survival. Development. 122:419-428.

24. Schmalbruch, H., Jensen, H.S., Bjaerg, M., Kamieniecka, Z., and Kurlan, L. 1991. A new mouse mutant with progressive motor neuronopathy. $J$. Neuropathol. Exp. Neurol. 50:192-204.

25. Gimenez y Ribotta, M., et al. 1997. Prevention of motoneuron death by adenovirus-mediated neurotrophic factors. J. Neurosci. Res. 48:281-285.

26. Gravel, G., Götz, R., Lorrain, A., and Sendtner, M. 1997. Adenoviral gene transfer of ciliary neurotrophic factor and brain-derived neurotrophic factor leads to long-term survival of axotomized motor neurons. Nat. Med. 3:765-770.

27. Baumgartner, B.J., and Shine, H.D. 1997. Targeted transduction of CNS neurons with adenoviral vectors carrying neurotrophic factor genes confers neuroprotection that exceeds the transduced population. J. Neurosci. 17:6504-6511.

28. Crouzet, J., et al. 1997. Recombinational construction in E. coli of infectious adenoviral genomes. Proc. Natl. Acad. Sci. USA. 94:1414-1419.

29. Hitt, M., Bett, A.J., Prevec, L., and Graham, F.L. 1994. Construction and propagation of human adenovirus vectors. In Cell biology - a laboratory handbook. Volume 1. J.E. Celis, editor. Academic Press. San Diego, CA. 479-490

30. Stratford-Perricaudet, L.D., Makeh, I., Perricaudet, M., and Briand, P. 1992. Widespread long-term gene transfer to mouse skeletal muscles and heart. J. Clin. Invest. 90:626-630.

31. Henderson, C.E., Bloch-Gallego, E., and Camu, W. 1995. Purified embryonic motoneurons. In Nerve cell culture: a practical approach. J. Cohen and G. Wilkin, editors. Oxford University Press. London, United Kingdom. 69-81.

32. Manthorpe, M., and Varon, S. 1989. Use of chick embryon ciliary ganglionic neurons for the in vitro assay of neurotrophic factors. In Dissection and tissue culture manual of the nervous system. A. Shahar, J. de Vellis, and A. Vernadakis, editors. Alan R. Liss. New York, NY. 317-321.

33. Haase, G., et al. 1999. Therapeutic benefit of CNTF in progressive motor neuronopathy depends on the route of administration. Ann. Neurol. 45:296-304.

34. Brunialti, A.L.B., Poirier, C., Schmalbruch, H., and Guenet, J.L. 1995. The mouse mutation progressive motor neuronopathy ( $p m n$ ) maps to chromosome 13. Genomics. 29:131-135.

35. Namba, T., Nakamura, T., and Grob, D. 1967. Staining for nerve fiber and cholinesterase activity in fresh frozen sections. Am. J. Clin. Pathol. 47:74-77.

36. Gurney, M.E., Yamamoto, H., and Kwon, Y. 1992. Induction of motor neuron sprouting in vivo by ciliary neurotrophic factor and basic fibroblast growth factor. J. Neurosci. 12:3241-3247

37. Oppenheim, R.W. 1986. The absence of significant postnatal motoneuron death in the brachial and lumbar spinal cord of rat. J. Comp. Neurol. 246:281-286

38. Park, S.H., Shub, C., Nobrega, T.P., Bailey, K.R., and Seward, J.B. 1996.
Two-dimensional echocardiographic calculation of left ventricular mass as recommended by the American Society of Echocardiography: correlation with autopsy and M-mode echocardiography. J. Am. Soc. Echocardiogr. 9:119-128.

39. Appel, S.H. 1981. A unifying hypothesis for the cause of amyotrophic lateral sclerosis, parkinsonism, and Alzheimer disease. Ann. Neurol. 10:499-505.

40. Sendtner, M., Götz, R., Holtmann, B., and Thoenen, H. 1997. Endogenous ciliary neurotrophic factor is a lesion factor for axotomized motoneurons in adult mice. J. Neurosci. 17:6999-7006.

41. Sagot, Y., et al. 1995. Polymer encapsulated cell lines genetically engineered to release ciliary neurotrophic factor can slow down progressive motor neuronopathy in the mouse. Eur. J. Neurosci. 7:1313-1322.

42. Arce, V., et al. 1998. Synergistic effects of Schwann- and muscle-derived factors on motoneuron survival involve GDNF and cardiotrophin-1 (CT1). J. Neurosci. 18:1440-1448

43. Ghadge, G.D., et al. 1995. CNS gene delivery by retrograde transport of recombinant replication-defective adenoviruses. Gene Ther. 2:132-137.

44. Baumgartner, B.J., and Shine, H.D. 1998. Neuroprotection of spinal motoneurons following targeted transduction with an adenoviral vector carrying the gene for glial cell line-derived neurotrophic factor. Exp. Neurol. 153:102-112.

45. Sagot, Y., Rossé, T., Vejsada, R., Perrelet, D., and Kato, A.C. 1998. Differential effects of neurotrophic factors on motoneuron retrograde labeling in a murine model of motoneuron disease. J. Neurosci. 18:1132-1141.

46. Sagot, Y., Tan, S.A., Hammang, J.P., Aebischer, P., and Kato, A.C. 1996. GDNF slows loss of motoneurons but not axonal degeneration or premature death of pmn mice. J. Neurosci. 16:2335-2341.

47. Haase, G., et al. 1998. Adenovirus-mediated transfer of the neurotrophin-3 gene into skeletal muscle of pmn mice: therapeutic effects and mechanisms of action. J. Neurol. Sci. 160(Suppl. 1):S97-S105.

48. Lewis, M.E., et al. 1993. The potential of insulin-like growth factor-I as a therapeutic for the treatment of neuromuscular disorders. Ann. NY Acad. Sci. 692:201-208.

49. Funakoshi, H., et al. 1995. Muscle-derived neurotrophin-4 as an activity-dependent trophic signal for adult motor neurons. Science. 268:1495-1499.

50. Henderson, J.T., Seniuk, N.A., Richardson, P.M., Gauldie, J., and Roder, J.C. 1994. Systemic administration of ciliary neurotrophic factor induces cachexia in rodents. J. Clin. Invest. 93:2632-2638.

51. Miller, R.G., et al. 1996. Toxicity and tolerability of recombinant human ciliary neurotrophic factor in patients with amyotrophic lateral sclerosis. Neurology. 47:1329-1331.

52. Akivama, Y., et al. 1997. In vivo effect of recombinant human leukemia inhibitory factor in primates. Jpn. J. Cancer. Res. 88:578-583.

53. Wollert, K.C., et al. 1996. Cardiotrophin-1 activates a distinct form of cardiac muscle cell hypertrophy. Assembly of sarcomeric units in series via gp130 leukemia inhibitory factor receptor-dependent pathways. $J$. Biol. Chem. 271:9535-9545. 